Delft University of Technology

\title{
Suppressed Charge Dispersion via Resonant Tunneling in a Single-Channel Transmon
}

Kringhøj, A.; Van Heck, B.; Larsen, T. W.; Erlandsson, O.; Sabonis, D.; Krogstrup, P.; Casparis, L.; Petersson, K. D.; Marcus, C. M.

DOI

10.1103/PhysRevLett.124.246803

Publication date

2020

Document Version

Final published version

Published in

Physical Review Letters

\section{Citation (APA)}

Kringhøj, A., Van Heck, B., Larsen, T. W., Erlandsson, O., Sabonis, D., Krogstrup, P., Casparis, L., Petersson, K. D., \& Marcus, C. M. (2020). Suppressed Charge Dispersion via Resonant Tunneling in a Single-Channel Transmon. Physical Review Letters, 124(24), [246803].

https://doi.org/10.1103/PhysRevLett.124.246803

Important note

To cite this publication, please use the final published version (if applicable).

Please check the document version above.

\section{Copyright}

Other than for strictly personal use, it is not permitted to download, forward or distribute the text or part of it, without the consent of the author(s) and/or copyright holder(s), unless the work is under an open content license such as Creative Commons.

\section{Takedown policy}

Please contact us and provide details if you believe this document breaches copyrights.

We will remove access to the work immediately and investigate your claim. 


\title{
Suppressed Charge Dispersion via Resonant Tunneling in a Single-Channel Transmon
}

\author{
A. Kringhøj®, ${ }^{1}$ B. van Heck, ${ }^{2,3}$ T. W. Larsen,,${ }^{1}$ O. Erlandsson $\odot,{ }^{1}$ D. Sabonis $\odot,{ }^{1}$ P. Krogstrup,${ }^{1,4}$ L. Casparis $\odot,{ }^{1}$ \\ K. D. Petersson ${ }^{1}{ }^{1}$ and C. M. Marcus ${ }^{1}$ \\ ${ }^{1}$ Microsoft Quantum Lab Copenhagen and Center for Quantum Devices, Niels Bohr Institute, University of Copenhagen, \\ Universitetsparken 5, 2100 Copenhagen, Denmark \\ ${ }^{2}$ Microsoft Quantum, Station Q, University of California, Santa Barbara, California 93106-6105, USA \\ ${ }^{3}$ Microsoft Quantum Lab Delft, Delft University of Technology, 2600 GA Delft. The Netherlands \\ ${ }^{4}$ Microsoft Quantum Materials Lab Copenhagen, Kanalvej 7, 2800 Lyngby, Denmark
}

(Received 22 November 2019; accepted 15 May 2020; published 19 June 2020)

\begin{abstract}
We demonstrate strong suppression of charge dispersion in a semiconductor-based transmon qubit across Josephson resonances associated with a quantum dot in the junction. On resonance, dispersion is drastically reduced compared to conventional transmons with corresponding Josephson and charging energies. We develop a model of qubit dispersion for a single-channel resonance, which is in quantitative agreement with experimental data.
\end{abstract}

DOI: 10.1103/PhysRevLett.124.246803

Superconducting circuits based on nonlinear Josephson junctions (JJs) form the basis of a broad array of coherent quantum devices used in applications ranging from radiation detectors to magnetometers to qubits [1,2]. An important application is the transmon qubit, a variant of the Cooper pair box qubit [3] where the Josephson energy $E_{J}$ of the junction exceeds the charging energy, $E_{C}=e^{2} / 2 C$, of the shunting capacitor with capacitance $C$. Designing qubits with ratio $E_{J} / E_{C}$ considerably greater than unity exponentially suppresses its charge character, correspondingly reducing its sensitivity to voltage noise and dramatically extending coherence $[4,5]$. The trade-off with increasing $E_{J} / E_{C}$ is reduced anharmonicity, which determines the minimal operation time due to leakage out of computational states [6].

The JJs used in superconducting qubits are almost exclusively based on superconductor-insulator-superconductor tunnel junctions [7], well described by a sinusoidal current-phase relation (CPR) [8]. More recently, gatevoltage-tunable transmon qubits (gatemons) have been realized using superconductor-semiconductor-superconductor (S-Sm-S) JJs, where the Sm weak link was either a nanowire [9,10], a two-dimensional electron gas [11], or graphene [12,13]. Such Sm weak links are typically quasiballistic and with Andreev processes [14] across the junction dominated by a small number of highly transmitting channels [15-17]. In this regime, the CPR is no longer sinusoidal, and anharmonicity deviates from the usual relations and trade-offs involving $E_{J}$ and $E_{C}$ [17].

An expected consequence of large transmission among a few Andreev modes in the JJ is a suppression of the quantization of island charge, which vanishes entirely when the transmission of any mode reaches unity [18-20]. Suppression of charge quantization in nonsuperconducting quantum dots has been well investigated experimentally [21,22], including a recent detailed study in a semiconductor quantum dot with vanishing level spacing due to an internal normal-metal contact [23]. In a similar fashion, charge quantization on a JJ-coupled superconducting island is expected to be suppressed for highly transmissive modes and vanish for unity transmission of a mode [24], irrespective of the ratio $E_{J} / E_{C}$, though to our knowledge this has not been previously investigated experimentally.

In this Letter, we investigate the charge dispersion in a nanowire-based gatemon qubit that shows strong suppression compared to a conventional metallic transmon qubit,
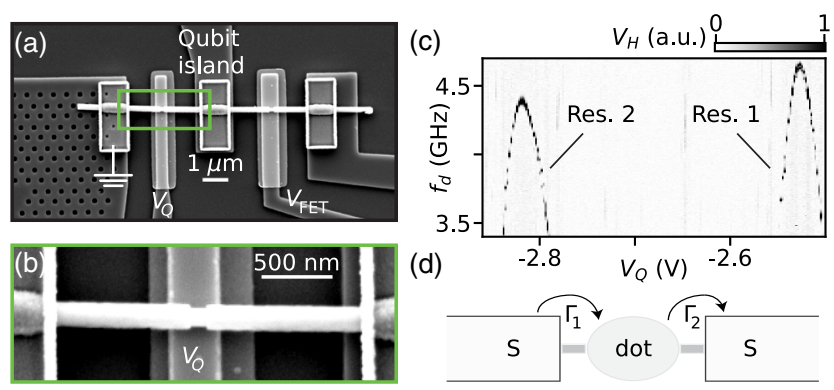

FIG. 1. (a) Scanning electron micrograph (SEM) of the nanowire region of the qubit device. Two etched regions were formed (qubit junction and FET) controlled with bottom gates $V_{Q}$ and $V_{\mathrm{FET}}$. (b) SEM of the qubit region highlighted (green square) in (a). (c) Two-tone spectroscopy measurements of the heterodyne transmission voltage $V_{H}$ at values of qubit gate voltage $V_{Q}$ just above complete depletion of the qubit junction and varying drive frequency $f_{d}$, yielding two resonances (Res. 1 and Res. 2) in the qubit frequency spectrum. (d) Sketch illustrating the principle of tunneling on and off a resonant dot level inside a Josephson junction connected to the superconducting leads by two tunnel barriers, characterized by tunnel rates $\Gamma_{1}$ and $\Gamma_{2}$. 
when operated across resonances in the junction. As discussed below, resonances in the semiconductor JJ effectively bring the Andreev transmission of a single mode close to unity. A comparison of experimental data to a simple model describing resonant Cooper pair transport across a single-mode junction [8,25-27] yields striking agreement, supporting both the general feature of suppressed charge quantization at large transmission and the additional feature that a dot resonance acts to provide an effective near-unity transmission of a single mode in a semiconductor JJ.

Measurements were performed on a gatemon qubit based on an InAs nanowire fully covered by $30 \mathrm{~nm}$ epitaxial $\mathrm{Al}$ [28], as described previously [29]. Two $\sim 150 \mathrm{~nm}$ segments of the $\mathrm{Al}$ shell were etched, forming gateable regions, as shown in Fig. 1(a), one serving as the qubit junction, controlled by gate voltage $V_{Q}$, and the other as a field-effect transistor (FET), allowing in situ dc transport, controlled by $V_{\text {FET }}$ [29]. All circuit QED measurements were carried out with the FET fully depleted $\left(V_{\mathrm{FET}}=-3 \mathrm{~V}\right)$, so that the gatemon circuit consisted of one side of the qubit junction contacted to ground and the other to the capacitor island [Fig. 1(b)]. The island capacitance was designed to yield $E_{C} / h \sim 500 \mathrm{MHz}$, allowing operation at intermediate $E_{J} / E_{C} \sim 10-20$ so that charge dispersion was easily resolved.

Near the pinch-off voltage of the qubit junction $\left(V_{Q} \sim-3 \mathrm{~V}\right)$, the first visible features to appear in twotone spectroscopy as $V_{Q}$ was tuned more positive were two narrow peaks in the qubit frequency, as shown in Fig. 1(c). We attribute these features to resonant tunneling of Cooper pairs through an accidental quantum dot formed in the junction [Fig. 1(d)], a common occurrence near full depletion $[30,31]$. We note that $\mathrm{dc}$ transport measurements (FET opened) of the switching current revealed corresponding resonances of similar width and spacing as a function of $V_{Q}$, supporting our interpretation of resonant tunneling across the junction (see Supplemental Material [32]).

To model the junction resonance, we consider a single spin-degenerate level at energy $\epsilon_{r}$, weakly coupled to the two superconducting leads via tunneling rates $\Gamma_{1}$ and $\Gamma_{2}$ [Fig. 2(a)] and a Breit-Wigner form for the transmission [33], $T=4 \Gamma_{1} \Gamma_{2} /\left(\epsilon_{r}^{2}+\Gamma^{2}\right)$, where $\Gamma=\Gamma_{1}+\Gamma_{2}$. Transmission is maximal on resonance, $\epsilon_{r}=0$, where it reaches unity for symmetric barriers, $\Gamma_{1}=\Gamma_{2}$ [Fig. 2(b)]. In the superconducting state, a pair of spin-degenerate Andreev bound states reside in the junction at energy $E$, given by $[8,26]$

$$
\begin{aligned}
& 2 \sqrt{\Delta^{2}-E^{2}} E^{2} \Gamma+\left(\Delta^{2}-E^{2}\right)\left(E^{2}-\epsilon_{r}^{2}-\Gamma^{2}\right) \\
& \quad+4 \Delta^{2} \Gamma_{1} \Gamma_{2} \sin ^{2}(\phi / 2)=0,
\end{aligned}
$$

where $\Delta$ is the superconducting gap and $\phi$ is the phase difference across the junction [32], as plotted in Fig. 2(c).
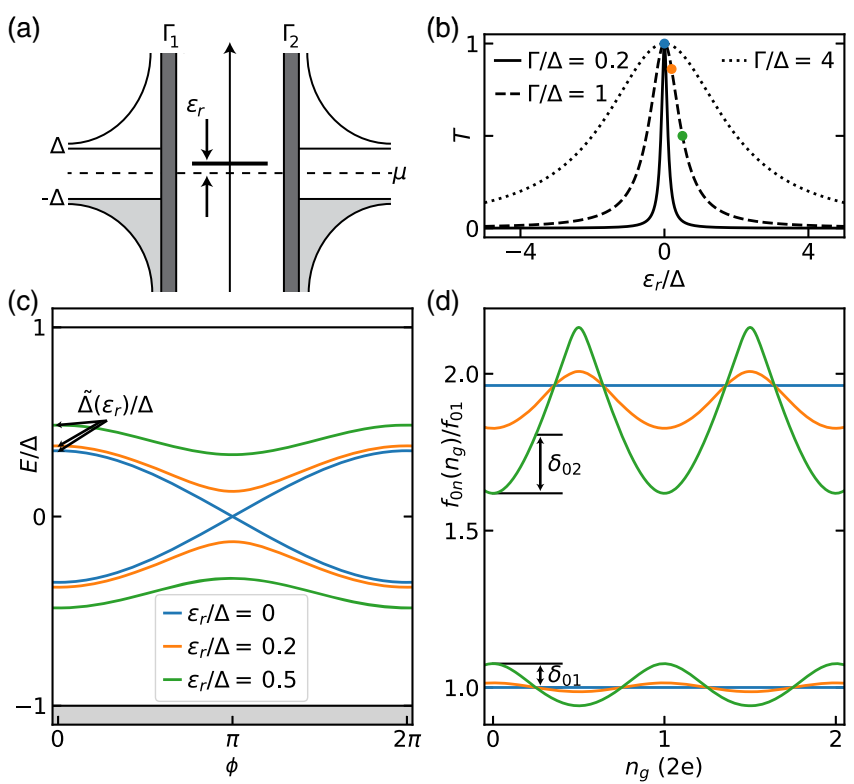

FIG. 2. (a) Sketch of the energy density of states of a superconductor-dot-superconductor system. The superconductors are described by a standard BCS density of states with gap $\Delta$. A spindegenerate level is located inside the JJ, detuned by $\epsilon_{r}$ from the Fermi level (dashed line). (b) Normal state transmission through the junction $T$ as a function of $\epsilon_{r}$ for three different $\Gamma$ for $\Gamma_{1}=\Gamma_{2}$. Note that $T=1$ occurs for $\epsilon_{r}=0$ for all $\Gamma$. (c) Numerical solutions to Eq. (1) describing resonant tunneling for three different $\epsilon_{r}$ [colored dots in (b)] and $\Gamma / \Delta=1$. The effective gap $\tilde{\Delta}\left(\epsilon_{r}\right)=E(0)$ (arrows) and continuum at $\pm E / \Delta=1$ (gray and white region) are indicated. (d) Numerical solutions to Eq. (2) showing the two lowest transition frequencies $f_{01}\left(n_{g}\right)$ and $f_{02}\left(n_{g}\right)$ as a function of offset charge $n_{g}$. The frequencies are normalized to the $0 \rightarrow 1$ degeneracy transition frequency $f_{01}(0.25)=f_{01}$ with dispersion amplitudes $\delta_{01}=f_{01}(0)-$ $f_{01}(0.25)$ and $\delta_{02}=f_{02}(0.25)-f_{02}(0)$ indicated (arrows).

The Andreev level spectrum consists of a spin-degenerate, phase-dependent bound state plus a continuum of quasiparticle states above the gap. At $\phi=0$, the bound state energy $E(0)=\tilde{\Delta}$, varies between $\epsilon_{r}$ and $\Delta$ as $\Gamma$ increases [32]. The energy gap at $\phi=\pi$ is proportional to the reflection amplitude $r=\sqrt{1-T}$ and thus vanishes at perfect transmission, yielding two decoupled $4 \pi$-periodic branches.

We model the charging-energy-induced quantum fluctuations in $\phi$ via the Hamiltonian [34-36],

$$
\begin{aligned}
H & =4 E_{C}\left(i \partial_{\phi}-n_{g}\right)^{2}+H_{J}, \\
H_{J} & =\tilde{\Delta}\left[\begin{array}{cc}
\cos (\phi / 2) & r \sin (\phi / 2) \\
r \sin (\phi / 2) & -\cos (\phi / 2)
\end{array}\right],
\end{aligned}
$$

where $n_{g}$ is the charge induced on the island in units of $2 e$. The model above was originally derived for a superconducting quantum point contact [35], and it is valid 
provided $E_{C} \ll \Delta$ and that the Andreev energies are well separated from the continuum. The eigenvalues of $H_{J}$,

$$
E= \pm \tilde{\Delta}\left[1-T \sin ^{2}(\phi / 2)\right]^{1 / 2},
$$

closely approximate the solutions of Eq. (1) (see Supplemental Material [32]). We solve Eq. (2) numerically [32] to obtain the qubit energy levels $E_{n}$ as well as the associated transition frequencies $f_{n m}\left(n_{g}\right)=\left[E_{m}\left(n_{g}\right)-\right.$ $\left.E_{n}\left(n_{g}\right)\right] / h$ [Fig. 2(d)].

A key feature of Eq. (2) is that it captures the dramatic effect of the presence of a level crossing at $\phi=\pi$ in the Andreev spectrum. At ideal transmission $(r=0)$, the two minima of the Josephson energy at $\phi=0$ and $2 \pi$ belong to two uncoupled branches of $H_{J}$, reflecting the fact that leftmoving Andreev states are uncoupled from right-moving Andreev states. As a consequence, the $2 \pi$ tunneling process is forbidden, and the charge dispersion reaches a minimal value dictated by the amplitude for $4 \pi$ tunneling [37]. The $2 \pi$ tunneling amplitude increases with $r$, since the two Andreev branches are coupled by backscattering. For a weakly transmitting channel, $r \gg\left(E_{c} / \tilde{\Delta}\right)$, it recovers to the known value corresponding to tunneling in a cosine potential. The remarkable flattening of the qubit energy levels at perfect transmission is illustrated in Fig. 2(d).

Measurements of charge dispersion across resonance 1 (Res. 1) in Fig. 1(c) were carried out by finely sweeping $V_{Q}$ while performing two-tone spectroscopy using a rastered drive tone $f_{d}$ followed by a readout tone at $f_{R} \sim$ $5.3 \mathrm{GHz}$ [Fig. 3(a)]. The fine sweep of $V_{Q}$ served two purposes: it both tuned the junction across the resonance and incremented the charge $n_{g}$ on the superconducting island, resulting in an oscillating pattern within a resonant envelope, appearing in the demodulated transmission voltage $V_{H}$ [Fig. 3(a)]. The two counteroscillating branches reflect fast quasiparticle poisoning of the island, which shifts the energy spectrum in Fig. 2(d) by half a period (1e) [5].

Qubit frequencies for both parity branches were extracted from the raw $V_{H}$ data using double Lorentzian fits for each $V_{Q}$, allowing determination of the maximal upper $\left(f_{+}\right)$and minimal lower $\left(f_{-}\right)$branch frequencies. At the charge degeneracy points, a single Lorentzian fit was used to find $f_{01}$. The charge dispersion amplitude, here defined $\delta_{01}=f_{+}-f_{01}$, was then extracted using an interpolated $f_{01}$ to determine $f_{+}$and $f_{01}$ at corresponding $V_{Q}$, as shown in Fig. 3(b). Near the top of the resonance, the two-photon transition frequency $f_{02}\left(n_{g}\right) / 2$ was visible in the spectrum and overlaps with the lower frequency branch of the $f_{01}$ transition [Fig. 3(c)]. As $\delta_{01}$ becomes comparable to the linewidth here we use the observed $f_{02}\left(n_{g}\right) / 2$ to identify the $V_{Q}$ associated with charge degeneracy and maximal dispersion amplitude.

Measurements of charge dispersion across Res. 2 were done in a slightly different way. Rather than using $V_{Q}$ to
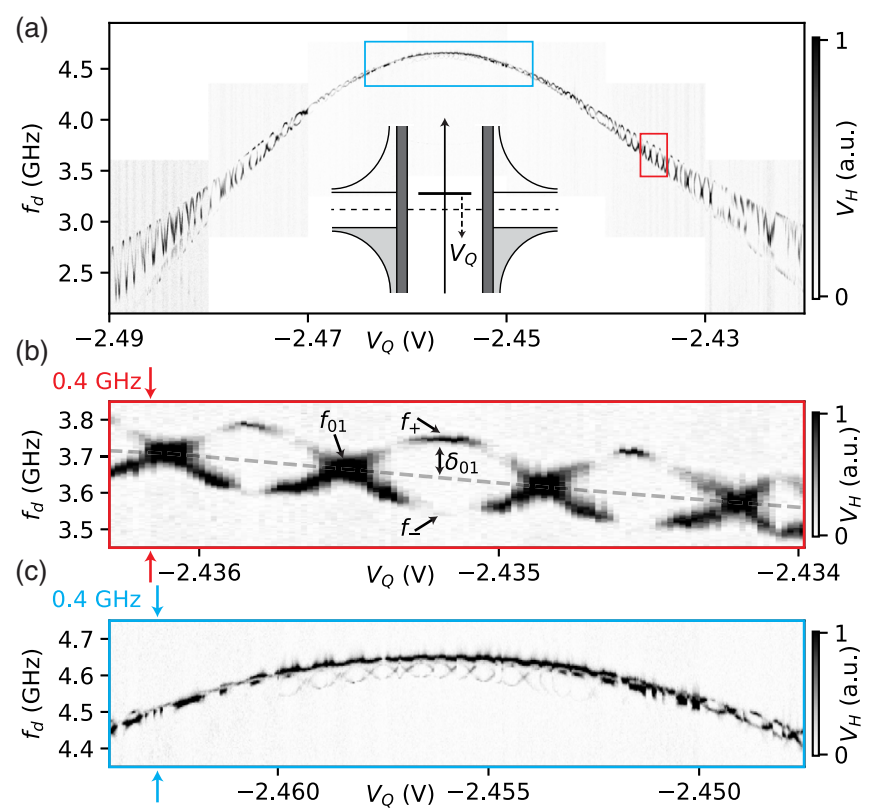

FIG. 3. (a) Measurement of the heterodyne transmission voltage $V_{H}$ as a function of $V_{Q}$ and a varying qubit drive $f_{d}$ across one of two resonances (Res. 1). (Inset) Sketch of the energy density of states to illustrate the interpretation that $\epsilon_{r}$ is varied by $V_{Q}$. (b),(c) Enlargement of the red (blue) region in (a) at the slope (peak) of the resonance spectrum. Note the same $f_{d}$ scale of $0.4 \mathrm{GHz}$ in both panels. Examples of maximal upper $\left(f_{+}\right)$, minimal lower $\left(f_{-}\right)$, and charge degeneracy $\left(f_{01}\right)$ frequencies are indicated in (b) (single arrows). An example of the maximal charge dispersion amplitude $\delta_{01}=f_{+}-f_{01}$ is indicated (double arrow). Interpolated $f_{01}$ as a function of $V_{Q}$ is shown in (b) (gray dashed line).

span the resonance and vary $n_{g}$, for Res. $2, n_{g}$ was varied by sweeping $V_{\mathrm{FET}}$ (in the depleted regime) at fixed $V_{Q}$ giving roughly independent control of $\epsilon_{r}$ and $n_{g}$ (see Supplemental Material [32]). The observed behavior of Res. 1 and Res. 2 was the same.

Figure 4 shows a parametric plot of dispersion $\delta_{01}$ as a function of $f_{01}$ for both resonances, with the original dependence of $f_{01}$ on $V_{Q}$ shown in the inset. As expected for transmons in general, $\delta_{01}$ decreases when $f_{01}$ increases due to an increase in $E_{J}$. In the $f_{01} \lesssim 3.5 \mathrm{GHz}$ range, corresponding to the tails of the two resonances, $\delta_{01}$ decays approximately exponentially as $f_{01}$ is increased. However, for the $f_{01} \gtrsim 4 \mathrm{GHz}$ range, near the top of the two resonances, we observe the onset of a sharper decrease toward vanishing $\delta_{01}$, strongly deviating from the exponential suppression expected in standard transmon qubits.

To quantitatively compare the observed charge dispersion across the resonances to the model (2), we first fix $\Delta=190 \mu \mathrm{eV}$ based on tunneling spectroscopy measurements at $V_{\mathrm{FET}}=+4 \mathrm{~V}$, where the FET is open [29]. For simplicity, we take the tunnel barriers to be symmetric and only allow $V_{Q}$ to tune $\epsilon_{r}$. We then fit $E_{C}$ (the same for both resonances) and $\Gamma$ (allowed to be different for each 


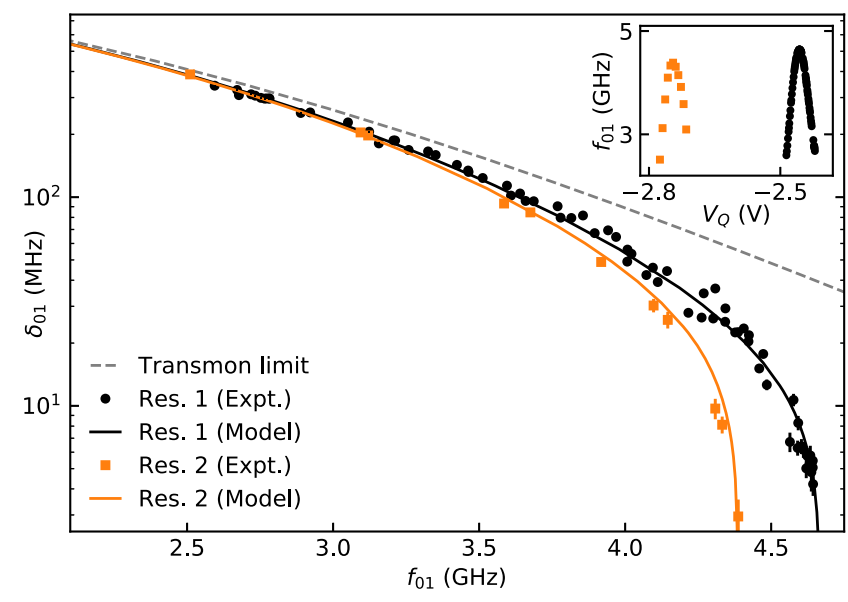

FIG. 4. Extracted maximal dispersion amplitudes (black and orange data points) and fit results (black and orange curves) of the $0 \rightarrow 1$ transition for both resonances (Res. 1 and Res. 2) as a function of qubit frequency $f_{01}$. The theory curves are fits of numerical solutions to Eq. (2) with fit parameters $E_{C} / h=$ $539 \mathrm{MHz}$ and $\Gamma / h=72(60) \mathrm{GHz}$ for Res. 1(2). Numerical $\delta_{01}$ (gray dashed line) for the standard transmon model with $E_{C} / h=539 \mathrm{MHz}$. Error bars are estimated from fit errors. (Inset) Extracted $f_{01}$ as a function of $V_{Q}$ for Res. 1 (black) and Res. 2 (orange).

resonance). Results are shown in Fig. 4, with $E_{C} / h=$ $539 \mathrm{MHz}$ (comparable to the electrostatic model [38] value $512 \mathrm{MHz}$ ) and $\Gamma / h=72 \mathrm{GHz}$ for Res. 1 , and $\Gamma / h=$ $60 \mathrm{GHz}$ for Res. 2.

Comparing $\delta_{01}$ to the prediction for a conventional transmon model based on the Hamiltonian $H_{T}=$ $4 E_{C}\left(n-n_{g}\right)^{2}-E_{J} \cos \phi$, for $E_{C} / h=539 \mathrm{MHz}$, highlights the suppressed dispersion observed experimentally and in the resonance model. The conventional model agrees with the experimental data and with the resonant level model only at low values of $f_{01}$, as expected for a decreasing transmission coefficient $(r \rightarrow 1)$, where the sinusoidal CPR is recovered.

When $V_{Q}$ is turned more positive, we no longer observed narrow, symmetric resonances associated with resonant tunneling. Instead, we observe a nonmonotonic spectrum much less susceptible to changes in $V_{Q}$. In this regime, we also observe a deviation in the charge dispersion compared to the value predicted by $H_{T}$ [32]. However, the suppression is not as pronounced as observed across the two resonances. We interpret this as crossing to a regime where the Andreev processes are no longer mediated by a resonant level and instead is described by a few gate tunable transmission coefficients [15-17,39], not reaching values similarly close to unity.

We also examine charge dispersion for the two-photon $(0 \rightarrow 2)$ transition frequencies of Res. 2. By increasing the power and repeating the scans used to extract $\delta_{01}$ we both excite the $0 \rightarrow 1$ and the $0 \rightarrow 2$ transitions. We define the $0 \rightarrow 2$ charge dispersion amplitude $\delta_{02}=f_{02}-f_{02,-}$,

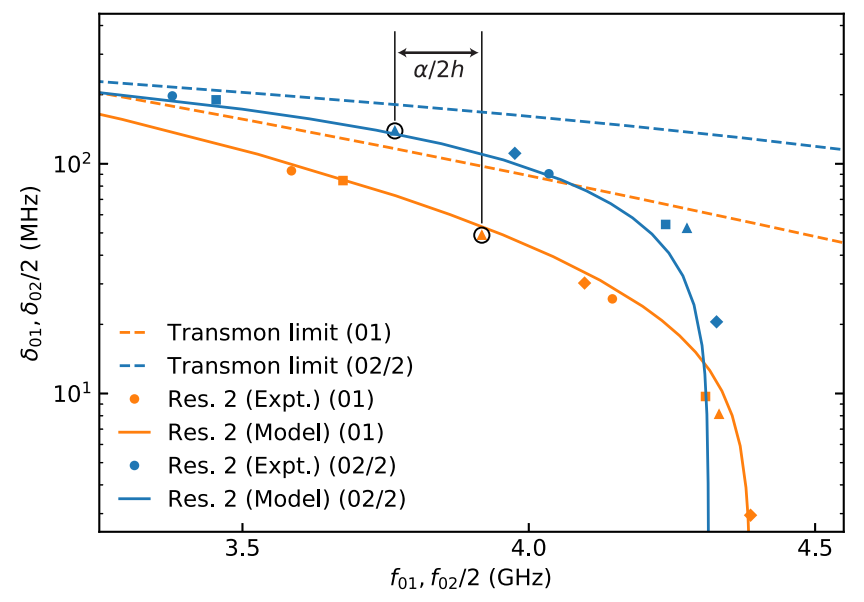

FIG. 5. Extracted maximal dispersion amplitudes (orange and blue data points) and fit result (orange and blue curves) of the $0 \rightarrow 1$ and $0 \rightarrow 2$ transitions of Res. 2 , respectively. The theory curves correspond to numerical solutions to Eq. (2) with $E_{C} / h=$ $539 \mathrm{MHz}$ and $\Gamma / h=60 \mathrm{GHz}$. Numerical $\delta_{01}$ (orange dashed line) and $\delta_{02} / 2$ (blue dashed line) based on $H_{T}$ with $E_{C} / h=539 \mathrm{MHz}$. The frequency differences between corresponding pairs of data points taken at same $V_{Q}$ (matching shapes) are equal to $\alpha / 2 h$, with one example indicated.

where $f_{02,-}$ and $f_{02}$ are the minimal lower branch and degeneracy frequency, respectively. This operative definition is chosen, as the upper branch of the $0 \rightarrow 2$ transition interferes with the lower branch of that of $0 \rightarrow 1$. Results for both $\delta_{01}$ and $\delta_{02} / 2$ are shown in Fig. 5. Both theory curves are obtained by solving Eq. (2) for the same parameters as in Fig. 4, again showing striking agreement between theory and experiment. We also compare the measured $\delta_{02} / 2$ with numerical solutions to $H_{T}$, again yielding roughly an order of magnitude deviation at resonance [40]. Finally, we emphasize that the finite frequency difference between the pairs of data points is equal to half the anharmonicity $\alpha$, as $f_{02} / 2-f_{01}=$ $1 / 2\left(f_{12}-f_{01}\right)=\alpha / 2 h$. This illustrates that $\delta_{0 i} \rightarrow 0$ can be achieved without $\alpha \rightarrow 0$ and, in principle, for much larger $\alpha$.

Minor deviations between experiment and model may be attributed to effects of electron-electron interactions in the quantum dot, which are not included in the model $[31,41,42]$ as well as fluctuations in the ratio $\Gamma_{1} / \Gamma_{2}$ as a function of $V_{Q}$.

In summary, we have observed and modeled the strong suppression of the charge dispersion in a single-channel transmon across a junction resonance, obtaining excellent agreement between experiment and theory. Our results suggest that charge dispersion can be suppressed without the necessity of large $E_{J} / E_{C}$ ratios. Future implementation of controlled dot structures or quantum point contact junctions to controllably achieve transmissions near unity may be a path to engineer superconducting qubits with vanishing charge dispersion and large anharmonicity. 
Additionally, a controllable near-unity junction would allow for deterministic tuning of the spectrum in Andreev qubits $[43,44]$. Parallel experiments demonstrate similar suppression of the charge dispersion in a half-shell nanowire transmon, including an investigation of qubit coherence times [45].

The numerical code and data accompanying the analysis of Figs. 4 and 5 are available online [40].

This work was supported by Microsoft and the Danish National Research Foundation. We acknowledge discussions with Karsten Flensberg, Michael Hell, and Martin Leijnse that inspired the ideas of the experiment. We thank Andrey Antipov, Arno Bargerbos, Gijs de Lange, Angela Kou, Roman Lutchyn, and Chaitanya Murthy for useful discussions. We acknowledge Marina Hesselberg, Karthik Jambunathan, Robert McNeil, Karolis Parfeniukas, Agnieszka Telecka, Shivendra Upadhyay, and Sachin Yadav for the device fabrication. B. v. H. thanks the Center for Quantum Devices, Niels Bohr Institute for the hospitality during part of the time in which this study was carried out. P. K. acknowledges funding from the European Research Commission through the Grant agreement "HEMs-DAM" No. 716655.

[1] Y. Makhlin, G. Schön, and A. Shnirman, Rev. Mod. Phys. 73, 357 (2001).

[2] S. M. Girvin, in Quantum Machines: Measurement and Control of Engineered Quantum Systems, Lecture Notes of the Les Houches Summer School Vol. 96 (Oxford University Press, New York, 2014).

[3] V. Bouchiat, D. Vion, P. Joyez, D. Esteve, and M. H. Devoret, Phys. Scr. T76, 165 (1998).

[4] J. Koch, T. M. Yu, J. M. Gambetta, A. A. Houck, D. I. Schuster, J. Majer, A. Blais, M. H. Devoret, S. M. Girvin, and R. J. Schoelkopf, Phys. Rev. A 76, 042319 (2007).

[5] J. A. Schreier, A. A. Houck, J. Koch, D. I. Schuster, B. R. Johnson, J. M. Chow, J. M. Gambetta, J. Majer, L. Frunzio, M. H. Devoret, S. M. Girvin, and R. J. Schoelkopf, Phys. Rev. B 77, 180502(R) (2008).

[6] P. Krantz, M. Kjaergaard, F. Yan, T. P. Orlando, S. Gustavsson, and W. D. Oliver, Appl. Phys. Rev. 6, 021318 (2019).

[7] H. Paik, D. I. Schuster, L. S. Bishop, G. Kirchmair, G. Catelani, A. P. Sears, B. R. Johnson, M. J. Reagor, L. Frunzio, L. I. Glazman, S. M. Girvin, M. H. Devoret, and R. J. Schoelkopf, Phys. Rev. Lett. 107, 240501 (2011).

[8] A. A. Golubov, M. Y. Kupriyanov, and E. Il'ichev, Rev. Mod. Phys. 76, 411 (2004).

[9] T. W. Larsen, K. D. Petersson, F. Kuemmeth, T. S. Jespersen, P. Krogstrup, J. Nygard, and C. M. Marcus, Phys. Rev. Lett. 115, 127001 (2015).

[10] G. de Lange, B. van Heck, A. Bruno, D. J. van Woerkom, A. Geresdi, S. R. Plissard, E. P. A. M. Bakkers, A. R. Akhmerov, and L. DiCarlo, Phys. Rev. Lett. 115, 127002 (2015).
[11] L. Casparis, M. R. Connolly, M. Kjaergaard, N. J. Pearson, A. Kringhøj, T. W. Larsen, F. Kuemmeth, T. Wang, C. Thomas, S. Gronin, G. C. Gardner, M. J. Manfra, C. M. Marcus, and K. D. Petersson, Nat. Nanotechnol. 13, 915 (2018).

[12] J. Kroll, W. Uilhoorn, K. van der Enden, D. de Jong, K. Watanabe, T. Taniguchi, S. Goswami, M. Cassidy, and L. Kouwenhoven, Nat. Commun. 9, 4615 (2018).

[13] J. I.-J. Wang, D. Rodan-Legrain, L. Bretheau, D. L. Campbell, B. Kannan, D. Kim, M. Kjaergaard, P. Krantz, G. O. Samach et al., Nat. Nanotechnol. 14, 120 (2019).

[14] C. W. J. Beenakker, Phys. Rev. Lett. 67, 3836 (1991).

[15] E. M. Spanton, M. T. Deng, S. Vaitiekènas, P. Krogstrup, J. Nygård, C. M. Marcus, and K. A. Moler, Nat. Phys. 13, 1177 (2017).

[16] M. F. Goffman, C. Urbina, H. Pothier, J. Nygård, C. M. Marcus, and P. Krogstrup, New J. Phys. 19, 092002 (2017).

[17] A. Kringhøj, L. Casparis, M. Hell, T. W. Larsen, F. Kuemmeth, M. Leijnse, K. Flensberg, P. Krogstrup, J. Nygård, K. D. Petersson, and C. M. Marcus, Phys. Rev. B 97, 060508(R) (2018).

[18] K. Flensberg, Phys. Rev. B 48, 11156 (1993).

[19] K. A. Matveev, Phys. Rev. B 51, 1743 (1995).

[20] Y. V. Nazarov, Phys. Rev. Lett. 82, 1245 (1999).

[21] S. R. Patel, S. M. Cronenwett, D. R. Stewart, A. G. Huibers, C. M. Marcus, C. I. Duruöz, J. S. Harris, K. Campman, and A. C. Gossard, Phys. Rev. Lett. 80, 4522 (1998).

[22] D. Duncan, D. Goldhaber-Gordon, R. Westervelt, K. Maranowski, and A. Gossard, Appl. Phys. Lett. 77, 2183 (2000).

[23] S. Jezouin, Z. Iftikhar, A. Anthore, F. D. Parmentier, U. Gennser, A. Cavanna, A. Ouerghi, I. P. Levkivskyi, E. Idrisov, E. V. Sukhorukov, L. I. Glazman, and F. Pierre, Nature (London) 536, 58 (2016).

[24] D. V. Averin, Phys. Rev. Lett. 82, 3685 (1999).

[25] L. Glazman and K. Matveev, JETP Lett. 49, 659 (1989), http://www.jetpletters.ac.ru/ps/1121/article_16988.shtml.

[26] C. W. J. Beenakker and H. van Houten, in Single-Electron Tunneling and Mesoscopic Devices, edited by H. Koch and H. Lübbig (Springer-Verlag, Berlin, 1992), pp. 175-179.

[27] I. A. Devyatov and M. Y. Kupriyanov, J. Exp. Theor. Phys. 85, 189 (1997).

[28] P. Krogstrup, N. L. B. Ziino, W. Chang, S. M. Albrecht, M. H. Madsen, E. Johnson, J. Nygård, C. M. Marcus, and T. S. Jespersen, Nat. Mater. 14, 400 (2015).

[29] A. Kringhøj, T. W. Larsen, B. van Heck, D. Sabonis, O. Erlandsson, I. Petkovic, D. I. Pikulin, P. Krogstrup, K. D. Petersson, and C. M. Marcus, Phys. Rev. Lett. 124, 056801 (2020).

[30] W. Chang, S. M. Albrecht, T. S. Jespersen, F. Kuemmeth, P. Krogstrup, J. Nygård, and C. M. Marcus, Nat. Nanotechnol. 10, 232 (2015).

[31] S. Hart, Z. Cui, G. Ménard, M. Deng, A. E. Antipov, R. M. Lutchyn, P. Krogstrup, C. M. Marcus, and K. A. Moler, Phys. Rev. B 100, 064523 (2019).

[32] See Supplemental Material at http://link.aps.org/ supplemental/10.1103/PhysRevLett.124.246803 for details on the theoretical modeling, transport measurements, data extraction, and open regime charge dispersion, which includes Refs. [8,24,26,29]. 
[33] A. Larkin and K. Matveev, JETP Lett. 66, 590 (1987), http:// www.jetp.ac.ru/cgi-bin/e/index/e/66/3/p580?a=list.

[34] D. A. Ivanov and M. V. Feigel'man, J. Exp. Theor. Phys. 87, 349 (1998).

[35] D. A. Ivanov and M. V. Feigel'man, Phys. Rev. B 59, 8444 (1999).

[36] A. Zazunov, V. S. Shumeiko, G. Wendin, and E. N. Bratus', Phys. Rev. B 71, 214505 (2005).

[37] Because of the large area below the potential barrier for $4 \pi$ tunneling at perfect transmission, this residual dispersion can be estimated to be well below the experimentally achieved linewidth.

[38] COMSOL, Inc., www.comsol.com.

[39] Y.-J. Doh, J. A. van Dam, A. L. Roest, E. P. A. M. Bakkers, L. P. Kouwenhoven, and S. De Franceschi, Science 309, 272 (2005).
[40] A. Kringhøj et al., Numerical code and data for: Suppressed Charge Dispersion via Resonant Tunneling in a SingleChannel Transmon, Version 1, Zenodo, (2020), https:// zenodo.org/record/3857794.

[41] E. Vecino, A. Martín-Rodero, and A. L. Yeyati, Phys. Rev. B 68, 035105 (2003).

[42] A. Martín-Rodero and A. L. Yeyati, Adv. Phys. 60, 899 (2011).

[43] C. Janvier, L. Tosi, L. Bretheau, Ç. Ö. Girit, M. Stern, P. Bertet, P. Joyez, D. Vion, D. Esteve, M. F. Goffman, H. Pothier, and C. Urbina, Science 349, 1199 (2015).

[44] M. Hays, G. de Lange, K. Serniak, D. J. van Woerkom, D. Bouman, P. Krogstrup, J. Nygård, A. Geresdi, and M. H. Devoret, Phys. Rev. Lett. 121, 047001 (2018).

[45] A. Bargerbos, W. Uilhoorn, C.-K. Yang, P. Krogstrup, L. P. Kouwenhoven, G. de Lange, B. van Heck, and A. Kou, preceding Letter, Phys. Rev. Lett. 124, 246802 (2020). 